\title{
Comments on the papers by Bierwisch and Zwicky
}

\author{
Wolfgang Klein
}

\section{MODULES AND INTERFACES}

The very notion of an interface presupposes the existence of clearly definable and separable components ("modules") between which this interface mediates. The components at issue here are syntax and morphology. Conventional wisdom tells us that morphology deals with the internal structure of words, and syntax with the way in which words are combined to larger units. Neither Zwicky nor Bierwisch cast any doubt on the principled nature of this distinction between morphology and syntax; but they provide us with numerous examples which might nurture such a doubt. Take Bierwisch's analysis of German verb clusters, according to which these clusters are formed by a "quasi-morphological process": "(...) complex verbs must be considered as boundary phenomena between syntax and morphology, such that verb cluster formation is only a quasi-morphological process" (p. 192). This means, among other things, that elements can be moved out of such a "complex word", as in (cf. Bierwisch's (67a)):
a. Er hätte ihn das Buch lesen sehen können
he had him the book read see can
"He would have been able to see him read the book"
b. Lesen sehen hätte er ihn das Buch können
read see had he him the book can
c. weil er ihn das Buch hätte lesen sehen können
because he him the book had read see can

In which sense, then, is an entity like "hat gehen lassen wollen" a word? Bierwisch lists a number of syntactic properties which it shares with simple verbs (i.e., words), whereas there are other properties which it shares with phrases. Could it be that there is simply no sharp distinction between a component called "morphology" and a component called "syntax"? And hence that "interface" is the wrong metaphor for what mediates between these two components? There is no shore line but an extended swamp between land and sea where the liquid and the solid are mixed. Not 


\section{W. Klein}

an attractive idea for a linguist. I will leave this point open for the moment and turn to Zwicky who, albeit more in passing, suggests a possible analysis of these constructions in his terms. An entity such as "hätte lesen sehen können" clearly violates Zwicky's "Intervention Constraint type III", hence it is not a "moreme", a morphological word in his sense. But it still could be a "syntactic word", a minimal entity on the token level, and in fact, this is the analysis suggested by Zwicky: "In all of these examples [including Bierwisch's "verb clusters", W.K.], what I am proposing is that some combination of material acts as a $\mathrm{W}$ syntactically. There is no necessary claim that such a combination instantiates a moreme; being word-like syntactically does not entail being word-like morphologically, though the standard situation is of course for a W to instantiate a moreme." (section 3.3). But this would only apply to the verb cluster in (c), whereas in (la) and in (lb), the "combination of material" is distributed over (minimally) two words. And even in the case of (1c), it remains open whether the entire cluster "hätte lesen sehen können" corresponds to a syntactic word, since Zwicky (quite understandably) gives no criteria for what should be understood by a "syntactic word in German".

The example illustrates a more general point. Whilst most linguists, including myself, would like to believe that there are two distinct and clearly separable components of grammar, "morphology" and "syntax", which interact at some point, closer inspection of even the most common constructions blurs this distinction, and hence the very notion of "modular organisation", and interfaces between modules, of grammar. Over the past years, numerous arguments have been given that complex words have an internal structure which parallels phrase structure in many ways (the contributions to this volume provide many examples). But what is then the difference between composition beneath the $\mathrm{X}^{0}$-level and above the $\mathrm{X}^{0}$-level? Is there any principled distinction at all? And still worse: What entitles us to call some compound entity " $\mathrm{X}^{0}$ ", rather than, for example, " $\mathrm{X}^{2 "}$ ? Why, for example, would most linguists say that the sequence of morphemes between determiner and auxiliary is a word in $(2 a)$, but rather some kind of phrase in $(2 b)$ :

$$
\begin{aligned}
& \text { a. Das Geworfen-Sein-in-das-Da ist unser Los } \\
& \text { The being-thrown-into-the-Here is our fate } \\
& \text { b. Das Werfen mit Steinen ist verboten } \\
& \text { The throwing with stones is forbidden }
\end{aligned}
$$

Examples of this type, and they are abundant, show that the criterion cannot be complexity. Nor can it be recursion, since mere is no rule-based end to compounds like "antidisestablishmentarianism" or, in German, which is notorious for these constructions, "Donaudampfschifffahrtsgesellschaftskapitän". 


\section{WORDS ARE NUTS}

Like a nut, a word may have a rich inner content; but the content is somehow encapsulated. The access to the structured interior is constrained. In particular, it is not selective: adjectives, adverbs or other modifying elements, when applied to a word, do not access just parts of the interior: they affect it as a whole. Bierwisch's case for the word-nature of German verb-clusters crucially hinges on this notion (see the argument in his section 2). Negation, for example, affects the entire cluster in much the same way in which it would affect a single word. (One might doubt this particular claim, since it is possible to limit the scope of the negation to one component, say "gehen" in (1), by giving it intonational prominence; but this does not affect the general point). Similarly, words have only limited "exits": Verbs, simple or compound, regularly have valencies, i.e., they require certain arguments around them. But these valencies come uniformly from the entire word, rather than from one of its components. Again, Bierwisch uses this as an argument for the wordhood of verb clusters: "a verb cluster created by functional composition behaves (...) like a single, but internally complex verb whose argument structure is derived from that of its constituent parts" (p.3). Much the same idea of words being somehow structured but encapsulated underlies Zwicky's various "Intervention constraints": Their common denominator is the fact that they all forbid some material to pierce the nutshell and to separate parts of its interior.

This notion of an "encapsulated structure" is compatible with the idea that the very same organisational principles obtain within the word and between words. What is constrained, are the relations from the structured interior of one word to the structured interior of another word.

Is such a view (which one would have to make more precise by defining the constraints on possible interrelations) correct? Probably not. A first problematic case are agglutinating languages, in which a "word" may simply embody all of the structural relations of an entire sentence, and parts of such a word can selectively be accessed by other words. But there are even elementary constructions in languages like English, for which such a neat separation between word-internal syntax ("morphology") and word-external syntax ("syntax" in the usual sense) is problematic. In what follows, I will briefly discuss such a case. Others are easily imaginable.

\section{POSTLEXICAL MORPHOLOGY}

In the following utterances
a. He seems to remember
b. He seemed to remember

the epistemic verb is marked for tense, the infinitive complement is not. Never- 


\section{W. Klein}

theless, we understand his remembering to be around the time of utterance TU in (3a), and before TU in (3b). Nothing in the infinitive complement itself tells us this. The infinitive "to remember" is neutral with respect to temporal reference. The time at which we understand the remembering to occur is somehow transferred from the time of the finite verb. Apparently, there is a general principle, which I will call "temporal congruency". It says that $\mathrm{T}(\mathrm{FIN})$, the time of the tensed verb, is transported to the verb in the infinitival complement. (It should be clear that this exposition of "temporal congruency" is a considerable simplification; for example, there are other verbs, such as "to promise", for which temporal congruency is different; but this does not concern our present argument). We may depict this as follows ("tc with [ ]" stands for "is temporally congruent with [ ]"):

$$
\text { c. T(FIN) tc with [ remember ] }
$$

If we want to express that the time of the finite verb falls into the posttime of the remembering, then we have to apply a "posttime operator" (PT) to the latter. In English (and similarly in Dutch, German, Latin and many other languages), this is done by perfect marking:

$$
\begin{aligned}
& \text { a. He seems to [have remembered] } \\
& \text { b. He seemed to [have remembered] }
\end{aligned}
$$

Temporal congruency then assigns "present" to [have remembered], i.e., to the posttime of remembering, in (4a); it means that one has right now the impression that he is in the posttime of remembering, hence was in the state of remembering at some earlier time. And in (4b), temporal congruency assigns "past" to [have remembered], i.e., the posttime of remembering: in the past, one had the impression that he was in the posttime of remembering; hence, the remembering itself was at some still earlier time. Again, we may depict the situation as follows:

$$
\text { c. T(FIN) tc with }[\mathrm{PT}(\text { remember })]
$$

The expression of PT in English combines morphological and syntactical devices inflection of the verb and the infinitival auxiliary "to have". One might argue that the latter is also "quasi-morphological". Bierwisch suggests this in the parallel case of German. But this is arguable in the case of English were a negation can intervene ("He seems to have never remembered"). We shall leave this open, since it is not really relevant to the point. (The very same argument could be made for Latin infinitival complements, such as "laboravisse videtur", which are clearly only morphological.)

The point is this: What happens if we expand the complement by a temporal adverbial? Adding such an adverb, like "yesterday", is clearly not morphological but a free syntactic operation: 
(5) He seems to have remembered yesterday

The question is now: Does T(yesterday) - the time expressed by "yesterday" specify the time of his remembering, or the posttime of his remembering? In other words, do we have $(6 a)$ or $(6 b)$ :
a. T(FIN) tc with
b. T(FIN) tc with [PT (yesterday (remember))]

Clearly, the latter is the case. It is not excluded that also part of the posttime falls into yesterday; but this is due to the particular example; if we narrow down the time of the temporal adverbial (for example by "yesterday at midday"), then reading (6a) is possible.

This means that the syntactic operation indeed pierces the nutshell: it has access not to the word "remembered" (or even "to have remembered") as a whole but to a selected part of its inner structure - it modifies "remember" - but not "remembered". Note that the latter possibility is not ruled out by semantic or by pragmatic reasons: the posttime of remembering is a time as well, and there is no reason why it should be impossible to specify this time by "yesterday" or by "yesterday at midday".

One straightforward way to describe these facts is to assume that morphological operations can be postlexical, i.e., apply after syntactic operations. In the above example, we may assume that first the syntactic operation "Add adverbial" applies to the verb "remember" and yields "remember yesterday". The posttime operator applies to the result of this syntactic operation and yields an inflected form. This gives us immediately the desired result.

The stipulation of postlexical morphology may seem to be a high price. But if we assume that there are ready-made lexical forms like "remembered" - or even "to have remembered" -, which are generated by lexicon-internal morphological processes and then passed on to syntax, then the price is much higher. It would force us to assume that common syntactic rules, such as the addition of temporal adverbial, operate into words, rather than on words. Furthermore, it would make constructions as common as "He seems to have remembered yesterday" non-compositional. And this seems even less desirable. 